\title{
MONOTONE INDEPENDENCE, COMB GRAPHS AND BOSE-EINSTEIN CONDENSATION
}

\author{
LUIGI ACCARDI \\ Università di Roma 2, Via Columbia 2, \\ I-00133 Rome, Italy \\ accardi@volterra.mat.uniroma2.it \\ ANIS BEN GHORBAL* \\ Institut für Mathematik und Informatik, \\ Ernst-Moritz-Arndt-Universität Greifswald, \\ Friedrich-Ludwig-Jahn-Straße 15a, \\ D-17487 Greifswald, Germany \\ ghorbal@uni-greifswald.de \\ NOBUAKI OBATA ${ }^{\dagger}$ \\ Graduate School of Information Sciences, \\ Tohoku University, Sendai 980-8579, Japan \\ obata@math.is.tohoku.ac.jp
}

Received 30 June 2003

Communicated by Y. G. Lu

\begin{abstract}
The adjacency matrix of a comb graph is decomposed into a sum of monotone independent random variables with respect to the vacuum state. The vacuum spectral distribution is shown to be asymptotically the arcsine law as a consequence of the monotone central limit theorem. As an example the comb lattice is studied with explicit calculation.

Keywords: Adjacency matrix; monotone independence; quantum decomposition; interacting Fock space; spectral distribution; comb graph; semicircle law.
\end{abstract}

AMS Subject Classification: 81S25, 82B20, 05E35, 60F05, 05C50

\section{Introduction}

The present paper combines three lines of research which have been objects of considerable attention in recent physical and mathematical literatures: (i) asymptotics

\footnotetext{
* Supported by the European Network Training Research "Quantum Probability with Applications to Physics, Information Theory and Biology".

†Supported in part by the program "RED support scheme for funding selected IT proposals" of the Ministry of Public Management, Home Affairs, Posts and Telecommunications.
} 
of large graphs, (ii) Bose-Einstein condensation (BEC), (iii) monotone independence.

The link between the first two lines was established in the papers by Burioni et $a l .{ }^{3,4}$ where it was proved that, in comb lattices, the phenomenon of BEC can also occur in dimension two, contrary to what happens in the usual Euclidean lattices. In other words, in analogy with general relativity, the effect of a complex geometry can be "... analogous to the introduction of an external confining potential ...". 4 We refer to Refs. 3 and 4 for more information on the physical motivations. To this picture we add the remark that, a particular class of geometries, the comb graphs which we introduce in this paper as a natural generalization of the comb lattices, are naturally related to monotone independence in the sense that the adjacency matrix of such graphs are decomposed into a sum of monotone independent random variables, and as a consequence of the monotone central limit theorem, its spectral distribution is asymptotically the arcsine law (Theorem 5.1).

This new concept of independence, introduced by $\mathrm{Lu},{ }^{7,22}$ and Muraki, ${ }^{24}$ occurs only beyond the classical (Kolmogorovian) probability theory. The theory of monotone probability has been developed by Muraki in several papers and parallels exactly to the analogue developments of classical, Bose, Fermi, Boolean, free, $q$-deformed, ... probability theories. Moreover, Muraki recently has proved that, by dropping the commutativity requirement from Schürmann's axiomatic characterization of independence, one arrives at exactly five possible notions of independence: Boolean, boson (including classical), free, monotone and antimonotone. ${ }^{28}$

Our results lead not only to some natural physical applications of monotone independence, the first known one to our knowledge, but also to an effective method for computing the asymptotic spectral distribution of the adjacency matrix. As a concrete illustration of our approach, we consider, in the second part of the present paper, the same comb lattice studied in Refs. 3 and 4 and we compute, using some general results due to Muraki, ${ }^{27}$ the exact vacuum distribution of the adjacency matrix for the infinite lattice. Our method applies directly to the infinite lattice, and therefore, differs from the method used in Refs. 3 and 4, which is based on thermodynamic limit with explicit calculation of the spectrum and is constrained to a specific model.

Our method also gives an explanation of the "hidden spectrum" discussed in Ref. 4. In fact we prove that the Hilbert space of the comb lattice is naturally decomposed into four sectors, invariant under the action of the adjacency matrix $A^{(2)}$ : in one of these invariant subspaces the spectral distribution of $A^{(2)}$ is the arcsine law on the interval $|x|<2 \sqrt{2}$ (Theorem 6.1); in another it is the semicircle distribution on the interval $|x|<2$ (Theorem 6.3); in the remaining two "offdiagonal" sectors, it is the monotone convolution of an arcsine and a semicircle laws whose density we determine explicitly (Theorem 6.2). The "hidden spectrum" of Sec. IV of Ref. 4 corresponds to the off-diagonal sectors and to the interval $[2,2 \sqrt{2}]$. 
The method we use to compute the spectral distribution of the adjacency matrix of a large graph has been developed during the last few years as a significant application of quantum (or, more generally, algebraic) probability theory. In particular, a key role is played by the relationship, pointed out by Accardi-Bożejko, ${ }^{2}$ between orthogonal polynomials and interacting Fock spaces. This new method has so far been applied to asymptotic spectral analysis of a growing family of graphs. A general theory is established by Hora-Obata ${ }^{17,18}$ after a series of explicit computations by Hashimoto, ${ }^{10}$ Hashimoto-Obata-Tabei, ${ }^{12}$ Hashimoto-Hora-Obata. ${ }^{11}$ However, except Hora's work ${ }^{15}$ on particular graphs whose vertices are Young diagrams, our discussion has been restricted only to regular graphs. The present paper shows that this new quantum probabilistic technique is very effective also for comb graphs that are not regular. Moreover, using the techniques developed by Hora, ${ }^{14,16}$ temperature dependence can be introduced and this will be discussed in a future paper.

\section{Adjacency Matrix}

Let $\mathcal{G}=(V, E)$ be a (non-oriented simple) graph, that is, $V$ is a non-empty set and $E \subset\{\{x, y\} ; x, y \in V, x \neq y\}$. Elements of $V$ and of $E$ are called a vertex and an edge, respectively. Two vertices $x, y \in V$ are called adjacent if $\{x, y\} \in E$, and in that case we also write $x \sim y$. The degree of $x \in V$, denoted by $\kappa(x)$, is by definition the number of vertices adjacent to $x$, namely,

$$
\kappa(x)=|\{y \in V ; y \sim x\}|,
$$

where $|\cdot|$ denotes the cardinality. A walk connecting two points $x, y \in V$ is a finite sequence of vertices such that $x \sim x_{1} \sim x_{2} \sim \cdots \sim x_{n-1} \sim y$. In this case $n$ is called the length of the walk. For two distinct vertices $x, y \in V$ the distance $d(x, y)$ is defined to be the shortest length of a walk connecting $x$ and $y$. By definition $d(x, x)=0$.

Throughout the paper we always assume that a graph is locally finite, i.e. $\kappa(x)<$ $\infty$ for all $x \in V$, and is connected, i.e. for any pair of vertices there exists a walk connecting them.

Given a graph $\mathcal{G}=(V, E)$, the adjacency matrix $A$ is defined by

$$
A_{x y}= \begin{cases}1, & \text { if } x \sim y, \\ 0, & \text { otherwise } .\end{cases}
$$

The graph structure is uniquely determined by the adjacency matrix. We consider $A$ as an operator acting on $\ell^{2}(V)$ from the left. For $x \in V$ let $\delta_{x}$ be the indicator function of a singlet $\{x\}$. Obviously, $\left\{\delta_{x} ; x \in V\right\}$ becomes a complete orthonormal basis of $\ell^{2}(V)$. The action of $\mathrm{A}$ is then uniquely determined by

$$
A \delta_{x}=\sum_{y \sim x} \delta_{y}, \quad x \in V .
$$

It is easily checked that $A$ is bounded if and only if $\sup _{x \in V} \kappa(x)<\infty$. 


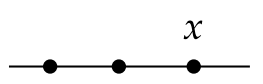

$G^{(1)}$

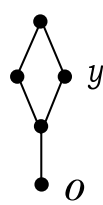

$G^{(2)}$

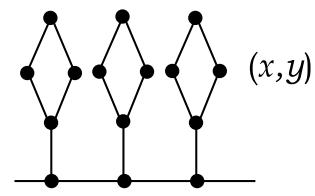

$G^{(1)} \triangleright_{0} G^{(2)}$

Fig. 1. Comb product.

\section{Comb Product}

Let $\mathcal{G}^{(1)}=\left(V^{(1)}, E^{(1)}\right)$ and $\mathcal{G}^{(2)}=\left(V^{(2)}, E^{(2)}\right)$ be two graphs. The comb product (or comb graph) of a backbone $\mathcal{G}^{(1)}$ and a finger $\mathcal{G}^{(2)}$ with a distinguished vertex $o \in V^{(2)}$ is by definition a graph obtained by grafting a copy of $\mathcal{G}^{(2)}$ at the vertex $o$ into each vertex of $\mathcal{G}^{(1)}$, see Fig. 1. This comb product is denoted by $\mathcal{G}^{(1)} \triangleright_{o} \mathcal{G}^{(2)}$.

By definition, the set of vertices of $\mathcal{G}^{(1)} \triangleright_{o} \mathcal{G}^{(2)}$ is identified with $V_{1} \times V_{2}$ and two vertices $(x, y),\left(x^{\prime}, y^{\prime}\right)$ are adjacent if and only if

(i) $x \sim x^{\prime}$ and $y=y^{\prime}=o$; or

(ii) $x=x^{\prime}$ and $y \sim y^{\prime}$.

Let $A^{(i)}$ be the adjacency matrix of $\mathcal{G}^{(i)}$. The adjacency matrix of the comb product $\mathcal{G}^{(1)} \triangleright_{o} \mathcal{G}^{(2)}$ is denoted by $A^{(1)} \triangleright_{o} A^{(2)}$.

Lemma 3.1. The matrix elements of $A^{(1)} \triangleright_{o} A^{(2)}$ are given by

$$
\left(A^{(1)} \triangleright_{o} A^{(2)}\right)_{(x, y),\left(x^{\prime}, y^{\prime}\right)}=A_{x x^{\prime}}^{(1)} \delta_{y o} \delta_{y^{\prime} o}+\delta_{x x^{\prime}} A_{y y^{\prime}}^{(2)},
$$

where $x, x^{\prime} \in V^{(1)}$ and $y, y^{\prime} \in V^{(2)}$.

Proof. A simple computation shows that

$$
A_{x x^{\prime}}^{(1)} \delta_{y o} \delta_{y^{\prime} o}+\delta_{x x^{\prime}} A_{y y^{\prime}}^{(2)}= \begin{cases}1, & \text { if } x \sim x^{\prime} \text { and } y=y^{\prime}=o, \\ 1, & \text { if } x=x^{\prime} \text { and } y \sim y^{\prime}, \\ 0, & \text { otherwise. }\end{cases}
$$

Then, in view of the conditions (i) and (ii) above, we see that the right-hand side of (3.1) takes value 1 if and only if $(x, y) \sim\left(x^{\prime}, y^{\prime}\right)$. In other words, the right-hand side of (3.1) coincides with the matrix element of the adjacency matrix of the comb product $\mathcal{G}^{(1)} \triangleright_{o} \mathcal{G}^{(2)}$.

Obviously, the comb product is not commutative; still it is associative.

Lemma 3.2. (Associativity) For $i=1,2,3$ let $\mathcal{G}^{(i)}$ be a graph with a distinguished vertex $o_{i}$. Then we have

$$
\left(\mathcal{G}^{(1)} \triangleright_{o_{2}} \mathcal{G}^{(2)}\right) \triangleright_{o_{3}} \mathcal{G}^{(3)}=\mathcal{G}^{(1)} \triangleright_{\left(o_{2}, o_{3}\right)}\left(\mathcal{G}^{(2)} \triangleright_{o_{3}} \mathcal{G}^{(3)}\right) .
$$


Proof. Straightforward from (3.1).

Whenever there is no danger of confusion, we omit the suffix $o$ and write $\mathcal{G}^{(1)} \triangleright$ $\mathcal{G}^{(2)}$ and $A^{(1)} \triangleright A^{(2)}$ for simplicity.

The adjacency matrix $A^{(1)} \triangleright A^{(2)}$ acts on $\ell^{2}\left(V_{1} \times V_{2}\right) \cong \ell^{2}\left(V_{1}\right) \otimes \ell^{2}\left(V_{2}\right)$. Let us examine this action in detail. We need notation. Let $P^{(2)}$ be the projection from $\ell^{2}\left(V_{2}\right)$ onto the one-dimensional subspace spanned by $\delta_{o}$, i.e. defined by

$$
P^{(2)} \psi(y)=\left\langle\delta_{o}, \psi\right\rangle \delta_{o}(y)=\delta_{y o} \psi(o), \quad \psi \in \ell^{2}\left(V_{2}\right) .
$$

Lemma 3.3. Notations being as above,

$$
A^{(1)} \triangleright A^{(2)}=A^{(1)} \otimes P^{(2)}+1 \otimes A^{(2)} .
$$

Proof. Let $\phi \in \ell^{2}\left(V_{1}\right)$ and $\psi \in \ell^{2}\left(V_{2}\right)$. Then, in view of (3.1) we have

$$
\begin{aligned}
\left(A^{(1)}\right. & \left.\triangleright A^{(2)}\right)(\phi \otimes \psi)(x, y) \\
& =\sum_{x^{\prime}, y^{\prime}}\left(A_{x x^{\prime}}^{(1)} \delta_{y o} \delta_{y^{\prime} o}+\delta_{x x^{\prime}} A_{y y^{\prime}}^{(2)}\right) \phi\left(x^{\prime}\right) \psi\left(y^{\prime}\right) \\
& =\sum_{x^{\prime}} A_{x x^{\prime}}^{(1)} \delta_{y o} \phi\left(x^{\prime}\right) \psi(o)+\sum_{y^{\prime}} A_{y y^{\prime}}^{(2)} \phi(x) \psi\left(y^{\prime}\right) \\
& =\left(A^{(1)} \phi\right)(x) \delta_{y o} \psi(o)+\phi(x)\left(A^{(2)} \psi\right)(y) .
\end{aligned}
$$

By (3.2) the first term becomes

$$
\left(A^{(1)} \phi\right)(x) \delta_{y o} \psi(o)=\left(A^{(1)} \phi\right)(x)\left(P^{(2)} \psi\right)(y)=\left(A^{(1)} \phi \otimes P^{(2)} \psi\right)(x, y) .
$$

On the other hand, the second term in (3.4) becomes

$$
\phi(x)\left(A^{(2)} \psi\right)(y)=\left(\phi \otimes A^{(2)} \psi\right)(x, y) .
$$

Then the assertion follows immediately.

Theorem 3.1. For $i=1,2, \ldots, n$ let $\mathcal{G}^{(i)}=\left(V^{(i)}, E^{(i)}\right)$ be a graph with a distinguished vertex $o_{i} \in V^{(i)}$. Then the adjacency matrix of the comb graph $\mathcal{G}^{(1)} \triangleright_{o_{2}} \mathcal{G}^{(2)} \triangleright_{o_{3}} \cdots \triangleright_{o_{n}} \mathcal{G}^{(n)}$ admits a decomposition of the form:

$$
A^{(1)} \triangleright A^{(2)} \triangleright \cdots \triangleright A^{(n)}=\sum_{i=1}^{n} \overbrace{1 \otimes \cdots \otimes 1}^{(i-1) \text { times }} \otimes A^{(i)} \otimes \overbrace{P^{(i+1)} \otimes \cdots \otimes P^{(n)}}^{(n-i) \text { times }},
$$

where $P^{(i)}$ is the projection from $\ell^{2}\left(V^{(i)}\right)$ onto the one-dimensional subspace spanned by $\delta_{o_{i}}$.

Proof. By induction from Lemmata 3.2 and 3.3. 


\section{Monotone Independence}

Definition 4.1. $\left(\right.$ Muraki $\left.^{26}\right)$ Let $(\mathcal{A}, \varphi)$ be an algebraic probability space. A family $\left\{X_{j} ; j \in T\right\}$ of elements of $\mathcal{A}$, where $T$ is a totally ordered set, is called monotone independent (with respect to $\varphi$ ) if

(i) for any choice of $i\langle j\rangle k, X_{i} X_{j}^{p} X_{k}=\varphi\left(X_{j}^{p}\right) X_{i} X_{k}$;

(ii) for any $m \geq 0, n \geq 0$ and for any $i_{m}>\cdots>i_{1}>i<j_{1}<\cdots<j_{n}$,

$$
\begin{aligned}
& \varphi\left(X_{i_{m}}^{p_{m}} \cdots X_{i_{1}}^{p_{1}} X_{i}^{r} X_{j_{1}}^{q_{1}} \cdots X_{j_{n}}^{q_{n}}\right) \\
& \quad=\varphi\left(X_{i_{m}}^{p_{m}}\right) \cdots \varphi\left(X_{i_{1}}^{p_{1}}\right) \varphi\left(X_{i}^{r}\right) \varphi\left(X_{j_{1}}^{q_{1}}\right) \cdots \varphi\left(X_{j_{n}}^{q_{n}}\right),
\end{aligned}
$$

where $p, p_{1}, \ldots, p_{m}, q_{1}, \ldots, q_{n}, r$ runs over the positive integers. Here $i\langle j\rangle k$ is a short hand notation of " $i<j$ and $j>k$ ". Similar convention is adopted also in condition (ii).

A concrete example of monotone independent random variables is given in the following

Proposition 4.1. For $i=1,2, \ldots, n$ let $\mathcal{H}^{(i)}$ be a Hilbert space equipped with a unit vector $\Phi^{(i)}$. Let $P^{(i)} \in \mathcal{B}\left(\mathcal{H}^{(i)}\right)$ be the projection onto the one-dimensional subspace spanned by $\Phi^{(i)}$. Then a sequence $\left\{X_{i}\right\}$ defined by

$$
X_{i}=\overbrace{1 \otimes \cdots \otimes 1}^{(i-1) \text { times }} \otimes B_{i} \otimes \overbrace{P^{(i+1)} \otimes \cdots \otimes P^{(n)}}^{(n-i)},
$$

where $B_{i} \in \mathcal{B}\left(\mathcal{H}^{(i)}\right)$, forms a monotone independent random variables with respect to the product state $\Phi^{(1)} \otimes \cdots \otimes \Phi^{(n)}$.

The proof is straightforward. This representation of monotone independent random variables was used by Liebscher in Ref. 20 and also in Franz's extension to monotone independence ${ }^{9}$ of Lenczewski's unification theorem ${ }^{19}$ see also Muraki ${ }^{27}$ for a more general form of monotone independent random variables.

A significant consequence of monotone independence is the monotone central limit theorem, which was proved by $\mathrm{Lu}^{22}$ and Muraki $^{24}$ with different motivations.

Theorem 4.1. (Monotone central limit theorem) Let $X_{1}, X_{2}, \ldots \in \mathcal{A}$ be a sequence of random variables of an algebraic probability space $(\mathcal{A}, \varphi)$ satisfying

(i) real, i.e. $X_{n}=X_{n}^{*}$;

(ii) monotone independent;

(iii) identically distributed, i.e. the moment sequence $\left\{\varphi\left(X_{n}^{m}\right)\right\}_{m=0}^{\infty}$ does not depend on the choice of $n$;

(iv) normalized as $\varphi\left(X_{n}\right)=0$ and $\varphi\left(X_{n}^{2}\right)=1$. 
Then, it holds that

$$
\lim _{n \rightarrow \infty} \varphi\left(\left(\frac{1}{\sqrt{n}} \sum_{k=1}^{n} X_{k}\right)^{m}\right)=\frac{1}{\pi} \int_{-\sqrt{2}}^{+\sqrt{2}} \frac{x^{m}}{\sqrt{2-x^{2}}} d x, \quad m=0,1,2, \ldots
$$

The probability distribution on the right-hand side of (4.1) is called the arcsine law normalized so as to have mean 0 and variance 1 .

\section{Asymptotic Spectrum of Comb Graphs}

For $i=1,2, \ldots, n$ let $\mathcal{G}^{(i)}=\left(V^{(i)}, E^{(i)}\right)$ be a graph with a distinguished vertex $o_{i} \in V^{(i)}$. Consider the Hilbert space

$$
\ell^{2}\left(V_{1} \times \cdots \times V_{n}\right) \cong \ell^{2}\left(V_{1}\right) \otimes \cdots \otimes \ell^{2}\left(V_{n}\right)
$$

equipped with a unit vector

$$
\Omega_{n}=\delta_{o_{1}} \otimes \delta_{o_{2}} \otimes \cdots \otimes \delta_{o_{n}}
$$

Let $\mathcal{B}_{n}$ be the $*$-algebra of bounded operators on the Hilbert space (5.1). Then the pair $\left(\mathcal{B}_{n}, \Omega_{n}\right)$ becomes an algebraic probability space.

Thus the adjacency matrix $A^{(1)} \triangleright_{o_{2}} A^{(2)} \triangleright_{o_{3}} \cdots \triangleright_{o_{n}} A^{(n)}$ is regarded as a real algebraic random variable of $\left(\mathcal{B}_{n}, \Omega_{n}\right)$. Moreover, it follows from Theorem 3.1 and Proposition 4.1 that the adjacency matrix is decomposed into a sum of monotone independent real random variables of $\left(\mathcal{B}_{n}, \Omega_{n}\right)$ :

$$
A^{(1)} \triangleright A^{(2)} \triangleright \cdots \triangleright A^{(n)}=\sum_{i=1}^{n} \overbrace{1 \otimes \cdots \otimes 1}^{(i-1) \text { times }} \otimes A^{(i)} \otimes \overbrace{P^{(i+1)} \otimes \cdots \otimes P^{(n)}}^{(n-i) \text { times }},
$$

where $P^{(i)}$ is the projection from $\ell^{2}\left(V^{(i)}\right)$ onto the one-dimensional subspace spanned by $\delta_{o_{i}}$.

Finally we come to the main assertion.

Theorem 5.1. Let $\mathcal{G}=(V, E)$ be a graph with a distinguished vertex $o \in V$. Consider a "homogenuous" comb product

$$
\mathcal{G}^{\triangleright n}=\mathcal{G} \triangleright_{o} \mathcal{G} \triangleright_{o} \cdots \triangleright_{o} \mathcal{G} \quad(n \text { times }),
$$

whose adjacency matrix is given by $A^{\triangleright n}=A \triangleright_{o} A \triangleright_{o} \cdots \triangleright_{o} A$ ( $n$ times $)$. Then it holds that

$$
\lim _{n \rightarrow \infty}\left\langle\Omega_{n},\left(\frac{A^{\triangleright n}}{\sqrt{n \kappa(o)}}\right)^{m} \Omega_{n}\right\rangle=\frac{1}{\pi} \int_{-\sqrt{2}}^{+\sqrt{2}} \frac{x^{m}}{\sqrt{2-x^{2}}} d x, \quad m=0,1,2, \ldots,
$$

where $\Omega_{n}=\delta_{o} \otimes \cdots \otimes \delta_{o}$ (n times) and $\kappa(o)$ is the degree of the distinguished vertex $o \in V$. 
Proof. Applying the decomposition (5.2) to the present case of homogeneous comb product, we have

$$
A^{\triangleright n}=\sum_{i=1}^{n} \overbrace{1 \otimes \cdots \otimes 1}^{(i-1) \text { times }} \otimes A \otimes \overbrace{P \otimes \cdots \otimes P}^{(n-i) \text { times }} \equiv \sum_{i=1}^{n} X_{i},
$$

where $P$ is the projection from $\ell^{2}(V)$ onto the one-dimensional subspace spanned by $\delta_{o}$. Obviously, $\left\{X_{i}\right\}$ satisfies conditions (i)-(iii) in Theorem 4.1. Moreover, since $\left\langle\delta_{o}, A \delta_{o}\right\rangle=0$,

$$
\left\langle\Omega_{n}, X_{i} \Omega_{n}\right\rangle=\prod_{j=1}^{i-1}\left\langle\delta_{o}, \delta_{o}\right\rangle \times\left\langle\delta_{o}, A \delta_{o}\right\rangle \times \prod_{j=i+1}^{n}\left\langle\delta_{o}, P \delta_{o}\right\rangle=0 .
$$

Similarly,

$$
\begin{aligned}
\left\langle\Omega_{n}, X_{i}^{2} \Omega_{n}\right\rangle & =\prod_{j=1}^{i-1}\left\langle\delta_{o}, \delta_{o}\right\rangle \times\left\langle\delta_{o}, A^{2} \delta_{o}\right\rangle \times \prod_{j=i+1}^{n}\left\langle\delta_{o}, P^{2} \delta_{o}\right\rangle \\
& =\left\langle\delta_{o}, A^{2} \delta_{o}\right\rangle=\left\langle A \delta_{o}, A \delta_{o}\right\rangle=\sum_{x \sim o} \sum_{y \sim o}\left\langle\delta_{x}, \delta_{y}\right\rangle=\kappa(o) .
\end{aligned}
$$

Hence $\left\{X_{i} / \sqrt{\kappa(o)}\right\}$ satisfies all the conditions in Theorem 4.1 and our assertion is a direct consequence of it.

\section{Comb Lattice}

\subsection{One-dimensional integer lattice}

We recall some elementary and noticeable facts on one-dimensional integer lattice $\mathbb{Z}$. By definition two points $i, j \in \mathbb{Z}$ are adjacent if and only if $|i-j|=1$. We take 0 as a distinguished vertex. The adjacency matrix of $\mathbb{Z}$ is denoted by $A$. The one dimensional integer lattice $\mathbb{Z}$ admits a natural stratification:

$$
\mathbb{Z}=\bigcup_{m=0}^{\infty} V_{m}, \quad V_{0}=\{0\}, \quad V_{m}=\{ \pm m\}, \quad m \geq 1
$$

Accordingly we define

$$
\Phi_{0}=\delta_{0}, \quad \Phi_{m}=\frac{1}{\sqrt{2}}\left(\delta_{m}+\delta_{-m}\right), \quad m \geq 1 .
$$

Then $\left\{\Phi_{m}\right\}_{m=0}^{\infty}$ is an orthonormal set in $\ell^{2}(\mathbb{Z})$ and we set

$$
\Gamma(\mathbb{Z})=\sum_{m=0}^{\infty} \oplus \mathbb{C} \Phi_{m}
$$

which is the closed subspace of $\ell^{2}(\mathbb{Z})$ generated by $\left\{\Phi_{m}\right\}_{m=0}^{\infty}$.

Proposition 6.1. $\Gamma(\mathbb{Z})$ is invariant under the action of $A$ and $\Phi_{0}$ is cyclic in $\Gamma(\mathbb{Z})$ under this action. 
Proof. By a direct computation we have

$$
\left\{\begin{array}{l}
A \Phi_{0}=\sqrt{2} \Phi_{1}, \\
A \Phi_{1}=\Phi_{2}+\sqrt{2} \Phi_{0}, \\
A \Phi_{m}=\Phi_{m+1}+\Phi_{m-1}, \quad m=2,3, \ldots,
\end{array}\right.
$$

from which the invariance of $\Gamma(\mathbb{Z})$ under the action of $A$ is clear. To prove the cyclicity of $\Phi_{0}$ under the action of $A$, let $R(A)$ denote the closed linear span of $\left\{A^{m} \Phi_{0} ; m=0,1,2, \ldots\right\}$. It is sufficient to show that $\Phi_{m} \in R(A)$ for all $m \geq 0$. Obviously, by (6.2) $\Phi_{1} \in R(A)$ and $\Phi_{2}=A \Phi_{1}-\sqrt{2} \Phi_{0} \in R(A)$. Suppose $m \geq 2$ and $\Phi_{1}, \Phi_{2}, \ldots, \Phi_{m} \in R(A)$. Then, from (6.2) we see that $\Phi_{m+1}=A \Phi_{m}-\Phi_{m-1} \in R(A)$. Hence the desired assertion follows by induction.

It is clear that (6.2) is a Jacobi type recurrence relation with Jacobi sequence

$$
\omega_{1}=2, \omega_{2}=\omega_{3}=\cdots=1 .
$$

Therefore there exists a unique interacting Fock structure in $\Gamma(\mathbb{Z})$ with creation and annihilation operators given by

$$
\begin{cases}B^{-} \Phi_{0}=0, \quad B^{-} \Phi_{m}=\sqrt{\omega_{m}} \Phi_{m-1}, & m \geq 1, \\ B^{+} \Phi_{m}=\sqrt{\omega_{m+1}} \Phi_{m+1}, & m \geq 0 .\end{cases}
$$

Proposition 6.2. It holds that

$$
\begin{aligned}
& A \uparrow_{\Gamma(\mathbb{Z})}=B^{+}+B^{-}, \\
& \left\|B^{ \pm}\right\| \leq \sqrt{2}, \quad\|A\| \leq 2 \sqrt{2},
\end{aligned}
$$

where $\|\cdot\|$ is the operator norm on $\Gamma(\mathbb{Z})$. Moreover, the probability distribution of $A$ in the vacuum state $\Phi_{0}$ is the arcsine law defined by

$$
\frac{d x}{\pi \sqrt{4-x^{2}}}, \quad|x|<2
$$

In other words,

$$
\left\langle\Phi_{0}, A^{m} \Phi_{0}\right\rangle=\frac{1}{\pi} \int_{-2}^{+2} \frac{x^{m}}{\sqrt{4-x^{2}}} d x, \quad m=0,1,2, \ldots
$$

Proof. (6.5) follows immediately by comparing (6.2) and (6.4). (6.6) follows from simple calculation: for $\xi=\sum_{m=0}^{\infty} \lambda_{m} \Phi_{m}$ we have

$$
\begin{aligned}
\left\|B^{-} \xi\right\|^{2} & =\left\|\sum_{m=0}^{\infty} \lambda_{m} B^{-} \Phi_{m}\right\|^{2}=\left\|\sum_{m=1}^{\infty} \lambda_{m} \sqrt{\omega_{m}} \Phi_{m-1}\right\|^{2} \\
& =\sum_{m=1}^{\infty}\left|\lambda_{m}\right|^{2} \omega_{m} \leq\left(\sup _{n} \omega_{n}\right)\|\xi\|^{2}
\end{aligned}
$$


from which $\left\|B^{-}\right\| \leq \sqrt{2}$ follows. Similarly, we have $\left\|B^{+}\right\| \leq \sqrt{2}$ and then $\|A\| \leq 2 \sqrt{2}$ is obvious.

We shall prove the second assertion. Let $\nu$ be the vacuum distribution of $A$. Since $\Phi_{0}$ is cyclic for $A$, it follows from the spectral theorem that $\Gamma(\mathbb{Z})$ is identified with $L^{2}(\mathbb{R}, \nu)$, where $A$ corresponds to the position operator $(A f(x)=x f(x))$. From the Accardi-Bożejko theorem ${ }^{2}$ we see that $\left\{\omega_{n}\right\}$ in (6.4) are precisely the Jacobi parameters of $\nu$. But it is known (and easy to prove directly) that the Jacobi sequence (6.3) uniquely characterizes the arcsine law as in (6.8).

Below we give a direct proof of the above fact not only as a nice application of the Cauchy-Stieltjes transform, but also beacuse this technique can be applied to more general cases when explicit formulaes are not available.

For (6.8) it is sufficient to compute $\left\langle\Phi_{0},\left(B^{+}+B^{-}\right)^{m} \Phi_{0}\right\rangle$. Let $\nu$ be the probability distribution determined by

$$
\left\langle\Phi_{0},\left(B^{+}+B^{-}\right)^{m} \Phi_{0}\right\rangle=\int_{-\infty}^{+\infty} x^{m} \nu(d x), \quad m=0,1,2, \ldots
$$

(Since $B^{ \pm}$are bounded operators, the support of $\nu$ is compact. Hence the moment problem is determinate and $\nu$ is uniquely determined.) Since $B^{ \pm}$is characterized by the Jacobi parameters $\left\{\omega_{n}\right\}$, so is $\nu$. In fact, the Cauchy-Stieltjes transform $G_{\nu}(z)$ admits a continued fraction expansion in terms of $\left\{\omega_{n}\right\}$. In our case $\left(\omega_{1}=2\right.$, $\omega_{2}=\omega_{3}=\ldots=1$ ), we have

$$
\int_{-\infty}^{+\infty} \frac{\nu(d x)}{z-x}=G_{\nu}(z)=\frac{1}{z}-\frac{\omega_{1}}{z}-\frac{\omega_{2}}{z}-\frac{\omega_{3}}{z}-\frac{\omega_{4}}{z}-\frac{\omega_{5}}{z}-\cdots=\frac{1}{\sqrt{z^{2}-4}} .
$$

Expanding both sides, we come to the following

$$
\begin{aligned}
\sum_{n=0}^{\infty} \frac{1}{z^{n+1}} \int_{-\infty}^{+\infty} x^{n} \nu(d x) & =\frac{1}{z} \sum_{n=0}^{\infty}\left(\begin{array}{c}
-1 / 2 \\
n
\end{array}\right)\left(-\frac{4}{z^{2}}\right)^{n} \\
& =\sum_{n=0}^{\infty}\left(\begin{array}{c}
-1 / 2 \\
n
\end{array}\right)(-1)^{n} \frac{4^{n}}{z^{2 n+1}} .
\end{aligned}
$$

Comparing the coefficients we get the moment sequence of $\nu$ as follows:

$$
\begin{aligned}
& \int_{-\infty}^{+\infty} x^{2 n+1} \nu(d x)=0 \\
& \quad \int_{-\infty}^{+\infty} x^{2 n} \nu(d x)=\left(\begin{array}{c}
-1 / 2 \\
n
\end{array}\right)(-1)^{n} 4^{n}=\frac{(2 n) !}{n ! n !}, \quad n=0,1,2, \ldots .
\end{aligned}
$$

Then we can conclude that $\nu$ coincides with the arcsine law (6.7). (Instead of calculating the moment sequence one may apply Stieltjes' inversion formula to $G_{\nu}(z)$ to obtain an explicit form of $\nu$.) 
Since $\Gamma(\mathbb{Z})$ is invariant under $A$, so is the orthogonal complement $\Gamma(\mathbb{Z})^{\perp}$. We set

$$
\Psi_{m}=\frac{1}{\sqrt{2}}\left(\delta_{m+1}-\delta_{-m-1}\right), \quad m=0,1,2, \ldots
$$

Then $\left\{\Psi_{m}\right\}$ forms an orthonormal basis of $\Gamma(\mathbb{Z})^{\perp}$, i.e.

$$
\Gamma(\mathbb{Z})^{\perp}=\sum_{m=0}^{\infty} \oplus \mathbb{C} \Psi_{m}
$$

By a direct computation we have

$$
A \Psi_{0}=\Psi_{1}, \quad A \Psi_{m}=\Psi_{m+1}+\Psi_{m-1}, \quad m=1,2, \ldots
$$

Repeating, for $\Gamma(\mathbb{Z})^{\perp}$, the same argument used for $\Gamma(\mathbb{Z})$ we arrive at the following:

Proposition 6.3. $\Gamma(\mathbb{Z})^{\perp}$ is invariant under $A$ and $\Psi_{0}$ is a cyclic vector. Moreover, $\Gamma(\mathbb{Z})^{\perp}$ is identified with the one mode free Fock space equipped with the annihilation and creation operators defined by

$$
\begin{cases}C^{-} \Psi_{0}=0, \quad C^{-} \Psi_{m}=\Psi_{m-1}, & m=1,2, \ldots, \\ C^{+} \Psi_{m}=\Psi_{m+1}, & m=0,1,2, \ldots\end{cases}
$$

In this identification it holds that

$$
A \uparrow_{\Gamma(\mathbb{Z})^{\perp}}=C^{+}+C^{-} .
$$

Hence

$$
\left\langle\Psi_{0}, A^{m} \Psi_{0}\right\rangle=\frac{1}{2 \pi} \int_{-2}^{+2} x^{m} \sqrt{4-x^{2}} d x, \quad m=0,1,2, \ldots,
$$

where the probability distribution on the right-hand side is Wigner's semicircle law.

Proof. (6.11) is immediate by comparing (6.9) and (6.10). Then (6.12) follows from the well-known fact that the Jacobi sequence of the semicircle law is $\left\{\omega_{n}=\right.$ $1\}$. (To obtain this result one may apply explicit computation as in the proof of Proposition 6.2.)

By general theory we know that the spectrum of $A \Gamma_{\Gamma(\mathbb{Z})}$ coincides with the support of the arcsine law (6.7) since $A$ admits a cyclic vector $\Phi_{0}$. A similar statement

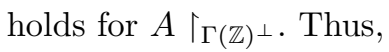

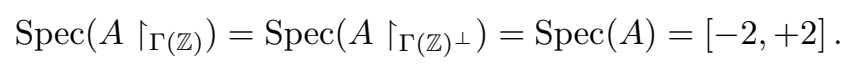

In fact, taking Propositions 6.2 and 6.3 into account, we have a canonical isomorphism:

$$
\begin{aligned}
\ell^{2}(\mathbb{Z}) & =\Gamma(\mathbb{Z}) \oplus \Gamma(\mathbb{Z})^{\perp} \\
& \cong L^{2}\left([-2,2], \frac{d x}{\pi \sqrt{4-x^{2}}}\right) \oplus L^{2}\left([-2,2], \frac{1}{2 \pi} \sqrt{4-x^{2}} d x\right),
\end{aligned}
$$


according to which the adjacency matrix $A$ is represented as $M_{x} \oplus M_{x}$, where $M_{x}$ is multiplication operator by $x$.

Remark 6.1. For $\alpha, \beta \in \mathbb{C}$ with $|\alpha|^{2}+|\beta|^{2}=1$, we consider a superposition of two states:

$$
\omega=\omega(\alpha, \beta)=\alpha \Phi_{0}+\beta \Psi_{0}=\alpha \delta_{0}+\frac{\beta}{\sqrt{2}}\left(\delta_{+1}-\delta_{-1}\right)
$$

Then

$$
\left\langle\omega, A^{m} \omega\right\rangle_{\ell^{2}(\mathbb{Z})}=\int_{-2}^{+2} x^{m} \rho_{\alpha, \beta}(x) d x, \quad m=0,1,2, \ldots
$$

where

$$
\rho_{\alpha, \beta}(x)=\frac{|\alpha|^{2}}{\pi \sqrt{4-x^{2}}}+\frac{|\beta|^{2}}{2 \pi} \sqrt{4-x^{2}} .
$$

Thus we obtain a one-parameter family of probability densities connecting the arcsine law and Wigner's semicircle law. This should be compared with the family of probability densities, constructed by Lu-Ruggieri, ${ }^{23}$ which also realize an interpolation between the arcsine and the semicircle law.

Remark 6.2. Let $A=A^{+}+A^{-}$be the quantum decomposition of the adjacency matrix $A$ according to the stratification (6.1), see Refs. 10 and 11 for generalities. Then both $\Gamma(\mathbb{Z})$ and $\Gamma(\mathbb{Z})^{\perp}$ are also invariant under the actions of $A^{ \pm}$and

$$
A^{ \pm} \Gamma_{\Gamma(\mathbb{Z})}=B^{ \pm}, \quad A^{ \pm} \uparrow_{\Gamma(\mathbb{Z})^{\perp}}=C^{ \pm} .
$$

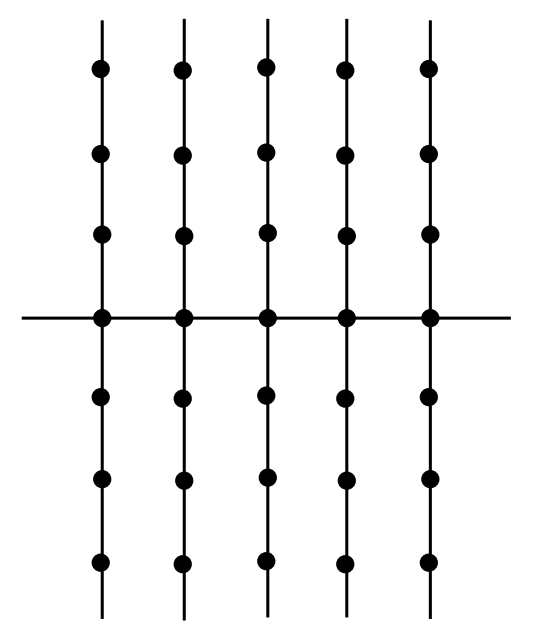

Fig. 2. Comb lattice $\mathbb{Z} \triangleright \mathbb{Z}$. 


\subsection{Comb lattice}

The comb product $\mathbb{Z} \triangleright_{0} \mathbb{Z}$ is called the two-dimensional comb lattice, see Fig. 2. The adjacency matrix is denoted by $A^{(2)}=A \triangleright_{0} A$ for simplicity. The vertices are given by $\mathbb{Z} \times \mathbb{Z}$. Then, $A^{(2)}$ acts on $\ell^{2}(\mathbb{Z} \times \mathbb{Z}) \cong \ell^{2}(\mathbb{Z}) \otimes \ell^{2}(\mathbb{Z})$ and, as is already shown in Sec. 3, we have

$$
A^{(2)}=A \otimes P+1 \otimes A
$$

where $P$ is the projection from $\ell^{2}(\mathbf{Z})$ onto the one-dimensional subspace spanned by $\delta_{0}=\Phi_{0}$.

Theorem 6.1. The distribution of the adjacency matrix $A^{(2)}$ in the vacuum state $\Phi_{0} \otimes \Phi_{0}$ is an arcsine law given by

$$
\frac{d x}{\pi \sqrt{8-x^{2}}}, \quad|x|<2 \sqrt{2}
$$

Namely,

$$
\left\langle\Phi_{0} \otimes \Phi_{0},\left(A^{(2)}\right)^{m} \Phi_{0} \otimes \Phi_{0}\right\rangle=\frac{1}{\pi} \int_{-2 \sqrt{2}}^{+2 \sqrt{2}} \frac{x^{m}}{\sqrt{8-x^{2}}} d x, \quad m=0,1,2, \ldots
$$

Proof. Let $\nu_{1}$ and $\nu_{2}$ be the distribution of $A \otimes P$ and $1 \otimes A$ in the vacuum state $\Phi_{0} \otimes \Phi_{0}$, respectively. As is easy to see, $\nu_{1}$ and $\nu_{2}$ coincide with the distribution of $A$ in $\Phi_{0}$, which is by Proposition 6.2 the arcsine law:

$$
\nu_{1}(d x)=\nu_{2}(d x)=\frac{d x}{\pi \sqrt{4-x^{2}}}, \quad|x|<2 .
$$

Since $A^{(2)}=A \otimes P+1 \otimes A$ is a sum of random variables which are monotone independent with respect to $\Phi_{0} \otimes \Phi_{0}$, see Sec. 5, the distribution $\mu$ of $A^{(2)}$ in $\Phi_{0} \otimes \Phi_{0}$ can be computed by Muraki's formula. ${ }^{27}$ Let $G_{\mu}$ be the Cauchy-Stieltjes transform of $\mu$ and put $H_{\mu}(z)=G_{\mu}(z)^{-1}$. Then Muraki's formula reads

$$
H_{\mu}(z)=H_{\nu_{1}}\left(H_{\nu_{2}}(z)\right)=\sqrt{H_{\nu_{2}}(z)^{2}-4}=\sqrt{z^{2}-8} .
$$

Hence

$$
G_{\mu}(z)=\frac{1}{\sqrt{z^{2}-8}} .
$$

By a similar computation as in the proof of Proposition 6.2 we obtain

$$
\begin{aligned}
\sum_{n=0}^{\infty} \frac{1}{z^{n+1}} \int_{-\infty}^{+\infty} x^{n} \mu(d x) & =\frac{1}{z} \sum_{n=0}^{\infty}\left(\begin{array}{c}
-1 / 2 \\
n
\end{array}\right)\left(-\frac{8}{z^{2}}\right)^{n} \\
& =\sum_{n=0}^{\infty}\left(\begin{array}{c}
-1 / 2 \\
n
\end{array}\right)(-1)^{n} \frac{8^{n}}{z^{2 n+1}} .
\end{aligned}
$$


Hence,

$$
\begin{aligned}
\int_{-\infty}^{+\infty} x^{2 n+1} \nu(d x) & =0 \\
\int_{-\infty}^{+\infty} x^{2 n} \nu(d x) & =\left(\begin{array}{c}
-1 / 2 \\
n
\end{array}\right)(-1)^{n} 8^{n}=\frac{2^{n}(2 n) !}{n ! n !}, \quad n=0,1,2, \ldots .
\end{aligned}
$$

On the other hand, we know that

$$
\int_{-2 \sqrt{2}}^{2 \sqrt{2}} \frac{x^{2 n}}{\pi \sqrt{8-x^{2}}} d x=\frac{2^{n}(2 n) !}{n ! n !}, \quad n=0,1,2, \ldots
$$

The assertion is then obvious.

Recall that in the orthogonal decomposition $\ell^{2}(\mathbb{Z})=\Gamma(\mathbb{Z}) \oplus \Gamma(\mathbb{Z})^{\perp}$ both subspaces are invariant under $A$ and $P$. Therefore the four subspaces obtaind from

$$
\begin{aligned}
\ell^{2}(\mathbb{Z}) \otimes \ell^{2}(\mathbb{Z})= & \left(\Gamma(\mathbb{Z}) \oplus \Gamma(\mathbb{Z})^{\perp}\right) \otimes\left(\Gamma(\mathbb{Z}) \oplus \Gamma(\mathbb{Z})^{\perp}\right) \\
= & (\Gamma(\mathbb{Z}) \otimes \Gamma(\mathbb{Z})) \oplus\left(\Gamma(\mathbb{Z})^{\perp} \otimes \Gamma(\mathbb{Z})\right) \\
& \oplus\left(\Gamma(\mathbb{Z}) \otimes \Gamma(\mathbb{Z})^{\perp}\right) \oplus\left(\Gamma(\mathbb{Z})^{\perp} \otimes \Gamma(\mathbb{Z})^{\perp}\right)
\end{aligned}
$$

are all invariant under $A^{(2)}$. In these spaces $\Phi_{0} \otimes \Phi_{0}, \Psi_{0} \otimes \Phi_{0}, \Phi_{0} \otimes \Psi_{0}, \Psi_{0} \otimes \Psi_{0}$ are regarded as vacuum vectors, respectively. In Theorem 6.1 the arcsine law with variance 2 is obtained from $\Phi_{0} \otimes \Phi_{0}$.

Theorem 6.2. The distribution of $A^{(2)}$ in $\Psi_{0} \otimes \Phi_{0}$ is

$$
\frac{1}{2 \pi}\left(\sqrt{8-x^{2}}-\sqrt{4-x^{2}} \chi_{[-2,+2]}(x)\right), \quad|x| \leq 2 \sqrt{2} .
$$

Proof. Let $\mu$ denote the distribution of $A^{(2)}$ in $\Psi_{0} \otimes \Phi_{0}$. From Proposition 4.1 we see that

$$
A^{(2)}=A \otimes P+1 \otimes A
$$

is also a sum of random variables which are monotone independent with respect to $\Psi_{0} \otimes \Phi_{0}$. Let $\nu_{1}$ and $\nu_{2}$ denote the distributions of $A \otimes P$ and $1 \otimes A$ in $\Psi_{0} \otimes \Phi_{0}$, respectively. We already know that $\nu_{1}$ is Wigner's semicircle law and $\nu_{2}$ is the arcsine law with variance 2. Hence, their reciprocal Cauchy-Stieltjes transforms are

$$
H_{\nu_{1}}(z)=\frac{2}{z-\sqrt{z^{2}-4}}, \quad H_{\nu_{2}}(z)=\sqrt{z^{2}-4} .
$$

Let $\mu$ denote the distribution of $A^{(2)}$ in $\Psi_{0} \otimes \Phi_{0}$. Applying Muraki's formula, we obtain

$$
H_{\mu}(z)=H_{\nu_{1}}\left(H_{\nu_{2}}(z)\right)=\frac{2}{\sqrt{z^{2}-4}-\sqrt{z^{2}-8}} .
$$


Hence the Cauchy-Stieltjes transform of $\mu$ is given by

$$
G_{\mu}(z)=\frac{1}{H_{\mu}(z)}=\frac{\sqrt{z^{2}-4}-\sqrt{z^{2}-8}}{2} .
$$

By virtue of Stieltjes' inversion formula we obtain the density function

$$
-\frac{1}{\pi} \lim _{y \downarrow 0} \operatorname{Im} G_{\mu}(x+i y)=\frac{1}{2 \pi}\left(\sqrt{8-x^{2}}-\sqrt{4-x^{2}} \chi_{[-2,+2]}(x)\right),
$$

as desired.

Theorem 6.3. The distributions of $A^{(2)}$ in $\Phi_{0} \otimes \Psi_{0}$ and $\Psi_{0} \otimes \Psi_{0}$ are Wigner's semicircle law:

$$
\frac{1}{2 \pi} \sqrt{4-x^{2}} d x, \quad|x| \leq 2
$$

Proof. Taking (6.13) into account, we have

$$
\left(A^{(2)}\right)^{m}=\sum_{k=0}^{m} A^{k} \otimes X(m-k, k),
$$

where $X(m-k, k)$ is the sum of $A^{m-k} P^{k}$ and its all possible permutations. Then,

$$
\left\langle\Phi_{0} \otimes \Psi_{0},\left(A^{(2)}\right)^{m} \Phi_{0} \otimes \Psi_{0}\right\rangle=\sum_{k=0}^{m}\left\langle\Phi_{0}, A^{k} \Phi_{0}\right\rangle\left\langle\Psi_{0}, X(m-k, k) \Psi_{0}\right\rangle .
$$

Since $\Psi_{0} \in \Gamma(\mathbb{Z})^{\perp}$ and $\Gamma(\mathbb{Z})^{\perp}$ is invariant under $A$ and $P, X(m-k, k) \Psi_{0} \in \Gamma(\mathbb{Z})^{\perp}$. Moreover, since $P$ acts on $\Gamma(\mathbb{Z})^{\perp}$ as zero operator, if $X(m-k, k)$ contains $P$, that is, if $1 \leq k \leq m$, we have $X(m-k, k) \Psi_{0}=0$. Thus, (6.14) becomes

$$
\left\langle\Phi_{0} \otimes \Psi_{0},\left(A^{(2)}\right)^{m} \Phi_{0} \otimes \Psi_{0}\right\rangle=\left\langle\Psi_{0}, X(m, 0) \Psi_{0}\right\rangle=\left\langle\Psi_{0}, A^{m} \Psi_{0}\right\rangle .
$$

Namely, the distribution of $A^{(2)}$ in $\Phi_{0} \otimes \Psi_{0}$ is the same as that of $A$ in $\Psi_{0}$, which is Wigner's semicircle law as is shown in Proposition 6.3. A similar argument yields the result for the distribution of $A^{(2)}$ in $\Psi_{0} \otimes \Psi_{0}$.

The distributions of $A^{(2)}$ in relevant states are summurized in the following table and the density functions are shown in Fig. 3.

\begin{tabular}{lcc}
\hline state & density function & support \\
\hline$\Phi_{0} \otimes \Phi_{0}$ & $\frac{1}{\pi \sqrt{8-x^{2}}}$ (arcsine law) & {$[-2 \sqrt{2},+2 \sqrt{2}]$} \\
$\Phi_{0} \otimes \Psi_{0}$ & $\frac{1}{2 \pi} \sqrt{4-x^{2}}$ (semicircle law) & {$[-2,+2]$} \\
$\Psi_{0} \otimes \Phi_{0}$ & $\frac{1}{2 \pi}\left(\sqrt{8-x^{2}}-\sqrt{4-x^{2}} \chi_{[-2,+2]}(x)\right)$ & {$[-2 \sqrt{2},+2 \sqrt{2}]$} \\
$\Psi_{0} \otimes \Psi_{0}$ & $\frac{1}{2 \pi} \sqrt{4-x^{2}}$ (semicircle law) & {$[-2,+2]$} \\
\hline
\end{tabular}




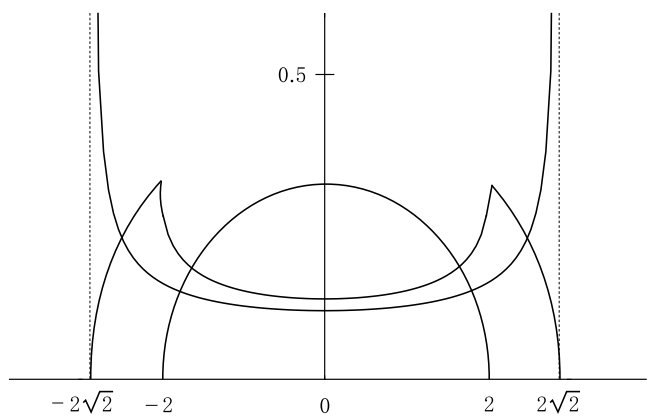

Fig. 3. Three distributions obtained from comb lattice $\mathbb{Z} \triangleright \mathbb{Z}$.

\section{Acknowledgments}

We would like to thank M. Schürmann and U. Franz for their stimulating discussion which was improved the presentation this paper.

\section{References}

1. L. Accardi, Y. G. Lu and I. Volovich, The QED Hilbert Module and Interacting Fock Spaces (Publications of IIAS, 1997).

2. L. Accardi, and M. Bożejko, Interacting Fock spaces and Gaussianization of probability measures, Infin. Dim. Anal. Quantum Probab. Rel. Topics 1 (1998) 663-670.

3. R. Burioni, D. Cassi, I. Meccoli, M. Rasetti, S. Regina, P. Sodano and A. Vezzani, Bose-Einstein condensation in inhomogenuous Josephson arrays, Europhys. Lett. 52 (2000) 251-256.

4. R. Burioni, D. Cassi, M. Rasetti, P. Sodano and A. Vezzani, Bose-Einstein condensation on inhomogenuous complex networks, J. Phys. B: At. Mol. Opt. Phys. 34 (2001) 4697-4710.

5. T. S. Chihara, An Introduction to Orthogonal Polynomials (Gordon and Breach, 1978).

6. M. de Giosa and Y. G. Lu, The free creation and annihilation operators as the central limit of quantum Bernoulli process, Random Oper. Stoch. Eq. 5 (1997) 227-236, preprint, Università di Bari, February (1995).

7. M. de Giosa and Y. G. Lu, From quantum Bernoulli process to creation and annihilation operators on interacting q-Fock space, Jpn. J. Math. 24 (1998) 149-167.

8. P. Deift, Orthogonal Polynomials and Random Matrices: A Riemann-Hilbert Approach, Courant Lect. Notes, Vol. 3 (Amer. Math. Soc., 1998).

9. U. Franz, Muraki's monotone independence is associative, Infin. Dim. Anal. Quantum Probab. Rel. Topics 4 (2001) 401-407.

10. Y. Hashimoto, Quantum decomposition in discrete groups and interacting Fock spaces, Infin. Dim. Anal. Quantum Probab. Rel. Topics 4 (2001) 277-287.

11. Y. Hashimoto, A. Hora and N. Obata, Central limit theorems for large graphs: Method of quantum decomposition, J. Math. Phys. 44 (2003) 71-88.

12. Y. Hashimoto, N. Obata and N. Tabei, A quantum aspect of asymptotic spectral analysis of large Hamming graphs, in Quantum Information III, eds. T. Hida and K. Saitô (World Scientific, 2001), pp. 45-57.

13. A. Hora, Central limit theorems and asymptotic spectral analysis on large graphs, Infin. Dim. Anal. Quantum Probab. Rel. Topics 1 (1998) 221-246. 
14. A. Hora, Gibbs state on a distance-regular graph and its application to a scaling limit of the spectral distributions of discrete Laplacians, Probab. Theory Rel. Fields $\mathbf{1 1 8}$ (2000) 115-130.

15. A. Hora, A noncommutative version of Kerov's Gaussian limit for the Plancherel measure of the symmetric group, in Asymptotic Combinatorics with Applications to Mathematical Physics, ed. A. M. Vershik Lect. Notes in Math. Vol. 1815 (SpringerVerlag, 2003), pp. 77-88.

16. A. Hora, Scaling limit for Gibbs states for Johnson graphs and resulting Meixner classes, Inf. Dim. Anal. Quantum Probab. Rel. Topics. 6 (2003) 139-143.

17. A. Hora and N. Obata, Quantum decomposition and quantum central limit theorem, in Fundamental Problems in Quantum Physics, eds. L. Accardi and S. Tasaki (World Scientific, 2003), pp. 284-305.

18. A. Hora and N. Obata, An interacting Fock space with periodic Jacobi parameter obtained from regular graphs in large scale limit, to appear in Quantum Information $V$, eds. T. Hida and K. Saitô (World Scientific).

19. R. Lenczewski, Unification of independence in quantum probability, Infin. Dim. Anal. Quantum Probab. Rel. Topics 1 (1998) 383-405.

20. V. Liebscher, On a central limit theorem for monotone noise, Infin. Dim. Anal. Quantum Probab. Rel. Topics 2 (1999) 155-167.

21. Y. G. Lu, An interacting free Fock space and the arcsine law, Probab. Math. Statist. 17 (1997) 149-166.

22. Y. G. Lu, On the interacting free Fock space and the deformed Wigner law, preprint Volterra No. 210 (1995), Nagoya Math. J. 145 (1997) 1-28.

23. Y. G. Lu and S. Ruggieri, A new example of interacting Fock space, to appear in Proc. First Sino-German Meeting on Stochastic Analysis, eds. S. Albeverio, Z. M. Ma and M. Röckner, Beijing 29 August-3 September 2002.

24. N. Muraki, A new example of noncommutative "de Moivre-Laplace theorem", in Probability Theory and Mathematical Statistics, eds. S. Watanabe, M. Fukushima, Yu. V. Prohorov and A. N. Shiryarev (World Scientific, 1996), pp. 353-362.

25. N. Muraki, Noncommutative Brownian motion in monotone Fock space, Commun. Math. Phys. 183 (1997) 557-570.

26. N. Muraki, Monotonic independence, monotonic central limit theorem and monotonic law of small numbers, Infin. Dim. Anal. Quantum Probab. Rel. Topics 4 (2001) 39-58.

27. N. Muraki, Monotonic convolution and monotonic Lévy-Hinčin formula, preprint, 2000 .

28. N. Muraki, The five independences as quasi-universal products, Infin. Dim. Anal. Quantum Probab. Rel. Topics 5 (2002) 113-134.

29. H. S. Wall, Analytic Theory of Continued Fractions (AMS Chelsea Pub., 1948). 